\title{
IRREDUCIBLE LOCAL SYSTEMS ON NILPOTENT ORBITS
}

\author{
ERIC N. SOMMERS \\ To George Lusztig, on the occasion of his 70th birthday \\ Department of Mathematics and Statistics, University of Massachusetts, Amherst, MA 01003, USA \\ E-mail: esommers@math.umass.edu \\ \|\|
}

Abstract

Let $G$ be a simple, simply-connected algebraic group over the complex numbers with Lie algebra $\mathfrak{g}$. The main result of this article is a proof that each irreducible representation of the fundamental group of the orbit $\mathcal{O}$ through a nilpotent element $e \in \mathfrak{g}$ lifts to a representation of a Jacobson-Morozov parabolic subgroup of $G$ associated to $e$. This result was shown in some cases by Barbasch and Vogan in their study of unipotent representations for complex groups and, in general, in an unpublished part of the author's doctoral thesis.

In the last section of the article, we state two applications of this result, whose details will appear elsewhere: to answering a question of Lusztig regarding special pieces in the exceptional groups (joint work with Fu, Juteau, and Levy); and to computing the $G$ module structure of the sections of an irreducible local system on $\mathcal{O}$. A key aspect of the latter application is some new cohomological statements that generalize those in earlier work of the author.

\section{Lifting Result}

Let $G$ be a simple, simply-connected algebraic group defined over the complex numbers $\mathbf{C}$ with Lie algebra $\mathfrak{g}$. Let $\mathcal{O}$ be a nilpotent orbit in $\mathfrak{g}$. Picking $e \in \mathcal{O}$, we can identify $\mathcal{O}$ with $G / G_{e}$, where $G_{e}=Z_{G}(e)$ is the centralizer in $G$ of $e$ under the adjoint action.

Received September 20, 2016 and in revised form October 18, 2016.

AMS Subject Classification: 17B08, 20G20, 20 G05.

Key words and phrases: Nilpotent orbits, orbit method, special pieces. 


\subsection{Statement of the result}

This paper is concerned with the irreducible representations

$$
\pi: G_{e} \rightarrow G L(V)
$$

such that the identity component $G_{e}^{\circ}$ of $G_{e}$ is in the kernel of $\pi$. In other words, $\pi$ descends to an irreducible representation of the component group $A(e):=G_{e} / G_{e}^{\circ}$. Since this group identifies with the fundamental group of $\mathcal{O}$ at the base point $e$ (in the analytic topology), we refer to $\pi$ or its associated bundle $G \times{ }^{G_{e}} V$ over $\mathcal{O}$ as an irreducible local system on $\mathcal{O}$.

Put $e$ in an $\mathfrak{s l}_{2}$-triple $\{e, h, f\}$ and write

$$
\mathfrak{g}=\bigoplus_{i \in \mathbb{Z}} \mathfrak{g}_{i}
$$

where $\mathfrak{g}_{i}$ is the $i$-eigenspace of $\operatorname{ad}(h)$ on $\mathfrak{g}$. Let $\mathfrak{p}=\oplus_{i \geq 0} \mathfrak{g}_{i}$. Let $P$ be the subgroup of $G$ with Lie algebra $\mathfrak{p}$ and let $L$ be the subgroup of $P$ with Lie algebra $\mathfrak{g}_{0}$. It is known that $G_{e} \subset P$ (see [2]). The main result of the paper is

Theorem 1.1. Let $(\pi, V)$ be an irreducible representation of $G_{e}$, trivial on $G_{e}^{\circ}$. Then there exists a representation $(\tilde{\pi}, V)$ of $P$ such that $\left.\tilde{\pi}\right|_{G_{e}}=\pi$.

Since $\pi$ is irreducible, any lifting $\tilde{\pi}$ to $P$ must also be irreducible. Therefore the unipotent radical $U_{P}$ of $P$ acts trivially on $V$ since the $U_{P}$-invariants are stable under the action of $P$ and must be nonzero. Thus $L$ must act irreducibly on $V$, and hence if $\tilde{\pi}$ exists, it can be specified by a highest weight representation $\lambda$ of $L$, after choosing a maximal torus $T$ in $L$ with $h \in \mathfrak{t}$ and a Borel subgroup $B$ with $T \subset B \subset P$, where $\mathfrak{t}$ is the Lie algebra of $T$.

In the classical groups, our proof is by direct construction using partitions; in the exceptional groups, we use the explicit knowledge of the structure of $A(e)$ from [11]. For applications it is also useful to find $\lambda \in \mathfrak{t}^{*}$ of minimal length, subject to a fixed $W$-invariant form on $\mathfrak{t}^{*}$.

For some orbits, Theorem 1.1 was proved by Barbasch and Vogan in [1, $\S 9]$ as part of their study of unitary representations for complex Lie groups. For the cases they consider there is a unified - and somewhat mysteriousexplanation for the highest weights that arise. 
Theorem 1.1 in full generality was proved in my $\mathrm{PhD}$ thesis [10] under the direction of George Lusztig. It was not published as it became part of a manuscript that dealt with the graded $G$-module of regular functions on the universal cover of $\mathcal{O}$. That manuscript (circa 2005) was distributed on a limited basis. In $\S 4.2$ we discuss some of the results from the manuscript. In $§ 4.1$ we give another application of the theorem to a question of Lusztig from [8] concerning special pieces in the exceptional groups, which is joint work in progress with Fu, Juteau, and Levy.

I thank David Vogan for many helpful discussions about Theorem 1.1 and its applications to rings of functions on orbit covers. I am deeply grateful to George Lusztig, for suggesting that Theorem 1.1 should hold in general, for his supervision of my thesis, and for his continued encouragement and friendship over the years.

\subsection{Algorithm for lifting}

We give a method to establish Theorem 1.1, which we carry out in the exceptional groups in the next section. Keep the notation from $\S 1.1$. Let $\mathfrak{m}$ denote the span of the $\mathfrak{s l}_{2}$-triple $\{e, h, f\}$ through $e$. Then $G_{e}=Z_{G}(\mathfrak{m}) U_{e}$ is a Levi decomposition, where $U_{e}$ is the unipotent radical of $G_{e}$. Now let $V_{\lambda}$ be a highest weight representation of $L$, viewed also as a representation of $P$ where $U$ acts trivially. Since $U_{e} \subset U$ and $Z_{G}(\mathfrak{m}) \subset L$, we are reduced to studying the restriction of $V_{\lambda}$ to the reductive group $Z_{G}(\mathfrak{m})$.

The first step in showing Theorem 1.1 is to see whether $G_{e}^{\circ}$ acts trivially on $V_{\lambda}$, which, by the above, is equivalent to $Z_{G}^{\circ}(\mathfrak{m})$ acting trivially. This condition is equivalent to a maximal torus $T^{\prime}$ of $Z_{G}(\mathfrak{m})$, and hence of $G_{e}^{\circ}$, acting trivially on $V_{\lambda}$, which is equivalent to each weight of $V_{\lambda}$ containing $T^{\prime}$ in its kernel.

To check this condition, we recall some results of Bala and Carter [2] and their generalization in [11], and we refer to those references for proofs. Let $W=N_{G}(T)$ be the Weyl group. Let $\Phi \subset \mathfrak{t}^{*}$ be the roots determined by $T$ and let $\Pi$ be the simple roots determined by $B$. Let $-\theta$ be the lowest root of $\Phi$ given the choice of $\Pi$. Set $\tilde{\Pi}=\Pi \cup\{-\theta\}$. Let $\mathcal{L}$ be the lattice 
of characters $X^{*}(T)$ of $T$. Given $J \subsetneq \tilde{\Pi}$, define $\mathcal{L}_{J}$ to be the $\mathbb{Z}$-lattice in $\mathcal{L}$ spanned by the elements of $J$. Set $\Phi_{J}=\mathcal{L}_{J} \cap \Phi$. Define the subalgebra

$$
\mathfrak{g}_{J}=\mathfrak{t} \oplus \bigoplus_{\alpha \in \Phi_{J}} \mathfrak{g}_{\alpha}
$$

where $\mathfrak{g}_{\alpha}$ is the root space for $\alpha$. Let $G_{J}$ denote the connected group in $G$ with Lie algebra $\mathfrak{g}_{J}$.

By Bala-Carter [2], there exists a pair $\left(\mathfrak{g}_{J}, e_{1}\right)$ with $J \subset \Pi$ and $e_{1} \in \mathfrak{g}_{J}$ distinguished nilpotent. Then the identity component of $T^{\mathcal{L}_{J}}$ is a maximal torus of $G_{e_{1}}$. Put $e_{1}$ in an $\mathfrak{s} l_{2}$-triple $\left\{e_{1}, h_{1}, f_{1}\right\}$ in $\mathfrak{g}_{J}$ with $h_{1} \in \mathfrak{t}$. Then there exists $w \in W$ such that $w\left(h_{1}\right)=h$ and it follows that $w\left(e_{1}\right) \in \mathfrak{g}_{2}$ (using $w$ also for any lift to $N_{G}(T)$ ) and so $w\left(e_{1}\right)=x \cdot e$ for some $x \in L$ since $L$ acts transitively on $G \cdot e \cap \mathfrak{g}_{2}$. Now $G_{e}^{\circ}$ acts trivially on $V_{\lambda}$ if and only if $G_{x \cdot e}^{\circ}=x\left(G_{e}^{\circ}\right) x^{-1}$ does. Hence it is enough to check that a maximal torus of $G_{w\left(e_{1}\right)}^{\circ}$ acts trivially on $V_{\lambda}$. Such a maximal torus is given by the identity component of $w\left(T^{\mathcal{L}_{J}}\right)$. Now a weight $\mu$ is trivial on the identity component of $w\left(T^{\mathcal{L}_{J}}\right)$ if and only if $n \cdot w^{-1}(\mu)$ is trivial on $T^{\mathcal{L}_{J}}$ for some positive integer $n$, which is equivalent to $n \cdot w^{-1}(\mu) \in \mathcal{L}_{J}$. Therefore,

Proposition 1.2. Let $\{e, h, f\}$ be an $\mathfrak{s} l_{2}$-triple used to define $P$ and $L$ with $h \in \mathfrak{t}$. Let $w \in W$ be such that $w^{-1}(h)$ is the semisimple part of an $\mathfrak{s} l_{2}$ triple $\left\{e_{1}, w^{-1}(h), f_{1}\right\}$ with $e_{1}$ distinguished in $\mathfrak{g}_{J}$ for $J \subset \Pi$. Then $G_{e}^{\circ}$ acts trivially on $V_{\lambda}$ if and only if each weight of $V_{\lambda}$ lies in the rational closure of $w \mathcal{L}_{J}$ in $X^{*}(T)$.

Example 1.3. Consider the nilpotent orbit of type $D_{4}\left(a_{1}\right)$ in $E_{6}$. Let $J=$ $\left\{\alpha_{2}, \alpha_{3}, \alpha_{4}, \alpha_{5}\right\}$, using Bourbaki's notation for labeling simple roots, so that $\mathfrak{g}_{J}$ has semisimple part of type $D_{4}$. Consider the semisimple element $h_{1} \in$ $\mathfrak{g}_{J} \cap \mathfrak{t}$ given by $4 \alpha_{3}^{\vee}+6 \alpha_{4}^{\vee}+4 \alpha_{5}^{\vee}+4 \alpha_{2}^{\vee}$ in the basis of simple coroots of $E_{6}$. Then $h_{1}$ completes to an $\mathfrak{s l}_{2}$-triple in $\mathfrak{g}_{J}$ whose nilpotent elements belong to $D_{4}\left(a_{1}\right)$. Applying $w=s_{4} s_{3} s_{5} s_{2} s_{4} s_{3} s_{5} s_{1} s_{6}$ where $s_{i}:=s_{\alpha_{i}}$ to $h_{1}$ yields a dominant element $h$ with values $\left\{\alpha_{i}(h)\right\}$ that give the weighted Dynkin diagram $00 \underset{0}{0} \underset{2}{2} 00$ of $D_{4}\left(a_{1}\right)$. Hence the Levi subgroup $L$ has semisimple type $A_{2}+A_{2}+A_{1}$ corresponding to all simple roots of $E_{6}$ except for $\alpha_{4}$.

The highest weight representation $V_{\varpi_{2}}$ of $L$ is two-dimensional with

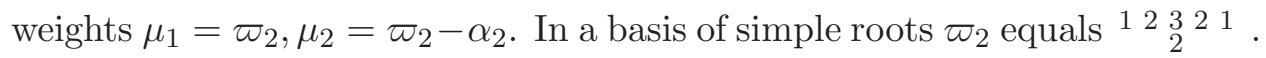

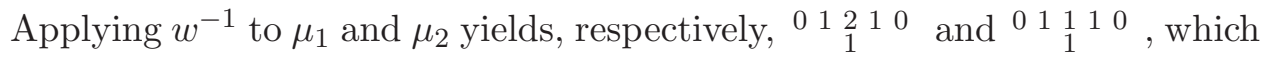


both lie in $\mathcal{L}_{J}$, the weights spanned by the roots of $J$. Hence $G_{e}^{\circ}$ acts trivially on $V_{\varpi_{2}}$.

Remark 1.4. All the representations in the proof of Theorem 1.1 turn out to be minuscule for $L$ and so the weights of $V_{\lambda}$ are a single orbit under the action of the Weyl group $W_{L}$ of $L$. Consequently only the highest weight $\lambda$ in Proposition 1.2 ends up needing to be checked.

For the rest of the section, assume $G$ is of adjoint type, so that $\mathcal{L}$ is generated by $\Phi$. We review some results from [11]. Define $c_{\alpha}$ by the equation $\theta=\sum_{\alpha \in \Pi} c_{\alpha} \alpha$ and set $c_{-\theta}=1$. Let

$$
d_{J}=\operatorname{gcd}\left(c_{\alpha}\right)_{\alpha \in \tilde{\Pi}-J}
$$

Since $G$ is adjoint, the torsion subgroup of $\mathcal{L} / \mathcal{L}_{J}$ is cyclic of order $d_{J}$ and is generated by the image of the element

$$
\tau_{J}=\frac{1}{d_{J}}\left(\sum_{\alpha \in \tilde{\Pi}-J} c_{\alpha} \alpha\right) .
$$

The center $Z\left(G_{J}\right)$ of $G_{J}$ equals $T^{\mathcal{L}_{J}}$ and so the character group of $Z\left(G_{J}\right)$ is isomorphic to $\mathcal{L} / \mathcal{L}_{J}$.

Let $C$ be a conjugacy class in $A(e)$. Then there exists a pair $\left(\mathfrak{g}_{J}, e_{1}\right)$ with $J \subsetneq \tilde{\Pi}$ and $e_{1} \in \mathfrak{g}_{J}$ a nilpotent element with the following properties: $e_{1}$ is distinguished in $\mathfrak{g}_{J} ; e=g \cdot e_{1}$ for some $g \in G$; and any $y \in Z\left(G_{J}\right)$ whose image generates $Z\left(G_{J}\right) / Z^{\circ}\left(G_{J}\right)$ has the property that the image of $g y g^{-1}$ in $A(e)$ lies in $C$. The trivial conjugacy class $C$ corresponds to the case where $J \subset \Pi$.

Now given that $V_{\lambda}$ is trivial on $G_{e}^{\circ}$ and thus descends to a representation of $A(e)$, we can describe how to compute the character of $V_{\lambda}$ as a representation of $A(e)$ on a conjugacy class $C \subset A(e)$. First, let $\left(\mathfrak{g}_{J}, e_{1}\right)$ be a pair as above corresponding to $C$. As before, put $e_{1}$ in an $\mathfrak{s} l_{2}$-triple $\left\{e_{1}, h_{1}, f_{1}\right\}$ in $\mathfrak{g}_{J}$ with $h_{1} \in \mathfrak{t}$ and let $w \in W$ be such that $w\left(h_{1}\right)=h$. Then as before $w\left(e_{1}\right)=x \cdot e$ for some $x \in L$. Next, choose $s \in T^{\mathcal{L}_{J}}$ so that $\tau_{J}(s)=\xi$, a primitive $d_{J}$-th root of unity. Then $s \in Z\left(G_{J}\right)$ and its image generates the component group of $Z\left(G_{J}\right)$. Hence, the image of $g s g^{-1}$ in $A(e)$ lies in $C$, where $g:=x^{-1} w$. 
Consequently the trace of an element of $C$ on $V_{\lambda}$ can be computed using the element $g s g^{-1}$ and this trace equals the trace of $w s w^{-1} \in T$ since $x \in L$. Let $\mu_{1}, \mu_{2}, \ldots$ be the not necessarily distinct weights of $V_{\lambda}$. Then the desired trace is given by $\sum \mu_{i}\left(w s w^{-1}\right)$, and this can be computed as follows. Since $\mu_{i}$ is trivial on any torus in $G_{w\left(e_{1}\right)}$ and since $T^{\mathcal{L}_{J}} \subset G_{e_{1}}$, it follows that $w^{-1}\left(\mu_{i}\right)$ is trivial on the identity component of $T^{\mathcal{L}_{J}}$. Hence the image of $w^{-1}\left(\mu_{i}\right)$ in $\mathcal{L} / \mathcal{L}_{J}$ is torsion and so must be an integral multiple $a_{i}$ of $\tau_{J}$, and so $\mu_{i}\left(w s w^{-1}\right)=\left(w^{-1} \mu_{i}\right)(s)=\xi^{a_{i}}$. Therefore,

Proposition 1.5. Let $\{e, h, f\}$ be as before. Let $w \in W$ be such that $w^{-1}(h)$ is the semisimple part of an $\mathfrak{s l}_{2}$-triple $\left\{e_{1}, w^{-1}(h), f_{1}\right\}$ with $e_{1}$ distinguished in $\mathfrak{g}_{J}$ for $J \subsetneq \tilde{\Pi}$. Let $\left\{\mu_{i}\right\}$ be the weights of $T$ on a representation $V_{\lambda}$ of $L$ where $G_{e}^{\circ}$ acts trivially. Then for each $i$ there exists $a_{i} \in \mathbb{Z}$ with $w^{-1}\left(\mu_{i}\right)=$ $a_{i} \tau_{J}$ modulo $\mathcal{L}_{J}$ and the trace on $V_{\lambda}$ of any element in the conjugacy class of $C$ parametrized by $\left(\mathfrak{g}_{J}, e_{1}\right)$ equals $\sum_{i} \xi^{a_{i}}$.

Example 1.6. We compute the character of $V_{\varpi_{2}}$ from the previous example on the conjugacy class parametrized by $3 A_{2}$ (the notation refers to the regular nilpotent element in the subalgebra of type $3 A_{2}$ ). Here $\tau_{J}=\alpha_{4}$. A semisimple element $h_{1}$ of an $\mathfrak{s l}_{2}$-triple for $e_{1}$ of type $3 A_{2}$ has weighted diagram in $E_{6}$ equal to $\begin{gathered}22-622 \\ 2\end{gathered}$ sends $h_{1}$ to $h$. Then $w^{-1} \mu_{1} \equiv-\tau_{J}$ and $w^{-1} \mu_{2} \equiv \tau_{J}$, modulo $\mathcal{L}_{J}$, and therefore the character value is $\xi^{-1}+\xi=-1$, where in this case $\xi$ is a primitive third root of unity. In a similar fashion, the character on the conjugacy class parametrized by $A_{3}+2 A_{1}$ is computed to be 0 and so $V_{\varpi_{2}}$ is the irreducible representation of $A(e) \simeq S_{3}$ of dimension two. In fact, the representations for this orbit fall under the framework in [1, $\S 9]$.

\section{Liftings in the Exceptional Groups}

2.1. We carried out the preceding algorithm for the exceptional groups to prove Theorem 1.1. Let $A^{\operatorname{simp}}(e)$ denote the component group relative to a simply-connected $G$ and let $A^{\text {adj }}(e)$ denote the component group relative to the adjoint group $G / Z(G)$. The information for the irreducible representations of $A^{\text {adj }}(e)$ are recorded in $\S 2.2$.

The algorithm begins by first running through the nonzero nodes of the weighted Dynkin diagram of $e$ to see if the corresponding one-dimensional 
representation $V_{\varpi}$ of $P$ descends to $A^{\text {simp }}(e)$. Remarkably this always happens in types $G_{2}, F_{4}, E_{7}, E_{8}$ (and later we will see it does in types $B, C, D$ ), even when $A^{\operatorname{simp}}(e)$ is trivial and even when $\varpi$ is not in the root lattice. Another way to say this is that $Z_{\mathfrak{g}}(\mathfrak{m})$, which we know belongs to $\mathfrak{l}$, actually belongs to $[\mathfrak{l}, \mathfrak{l}]$. In many cases, this is true because $Z_{\mathfrak{g}}(\mathfrak{m})$ is semisimple, but in the remaining cases it seems surprising. This fact is related to the observation by several researchers that in the above cases the number of irreducible components of codimension one of the complement of $P \cdot e$ in $\mathfrak{g}_{2}$ is the number of nonzero nodes of the weighted Dynkin diagram. In type $E_{6}$ the same fact holds whenever $A^{\operatorname{simp}}(e)$ is non-trivial even when $\varpi$ is not in the root lattice. Consequently, we omit the trivial representation from our tables since we get a trivial representation of $A^{\operatorname{simp}}(e)$ for each node of the weighted Dynkin diagram with nonzero value that does not yield a non-trivial representation of $A^{\operatorname{simp}}(e)$.

We also checked in $E_{6}$ (respectively, $E_{7}$ ) that if $\varpi$ is a fundamental weight not in the root lattice and $\varpi$ corresponds to a node with non-zero value in the weighted Dynkin diagram, then $V_{3 \varpi}$ (respectively, $V_{2 \varpi}$ ) descends to a trivial representation of $A^{\text {adj }}(e)$. This implies that the kernel of the representation $V_{\varpi}$, viewed as a representation of $A^{\operatorname{simp}}(e)$, is a normal subgroup of $A^{\operatorname{simp}}(e)$ isomorphic to $A^{\text {adj }}(e)$. Hence $A^{\operatorname{simp}}(e)$ must be a split central extension of $A^{\text {adj }}(e)$. Furthermore, we observe that whenever $\left|A^{\operatorname{simp}}(e)\right|>\left|A^{\operatorname{adj}}(e)\right|$, there is always one such node in the weighted Dynkin diagram. This implies that $A^{\operatorname{simp}}(e)$ is always a split central extension of $A^{\text {adj }}(e)$ in $E_{6}$ and $E_{7}$, which gives a new proof of the splitting in these cases (we note that there is no splitting in general in types $B$ and $D$, see $\S 3.2$ ). It follows that by tensoring the one-dimensional representations coming from these fundamental weights (which are trivial on $A^{\text {adj }}(e)$ ) with the representations that we found for $A^{\text {adj }}(e)$ (which are trivial on the image of the center of $G$ ), all the irreducible representations for $A^{\operatorname{simp}}(e)$ in $E_{6}$ and $E_{7}$ are obtained. This completes the proof of Theorem 1.1 in the exceptional groups.

\subsection{Tables}

In the following tables we list weights that yield nontrivial irreducible representations of $A^{\text {adj }}(e)$ in the exceptional groups. From the discussion 
in the previous section, this allows us to find all weights of $L$ that give rise to irreducible representations of $A^{\operatorname{simp}}(e)$. From there, it is easy enough to construct lifts of minimal length. The numbering of simple roots is given in types $E$ by ${ }^{13} \frac{4}{2} \ldots n$ as in Bourbaki (but different from that in [10]). We first list the cases where $A^{\text {adj }}(e) \simeq S_{2}$.

\begin{tabular}{|cccc|c|c|}
\hline \multicolumn{6}{|c|}{$F_{4}$} \\
\hline & $\cdots$ & Bala-Carter & Sign rep \\
\hline 0 & 0 & 0 & 1 & $\tilde{A}_{1}$ & $\varpi_{4}$ \\
\hline 2 & 0 & 0 & 0 & $A_{2}$ & $\varpi_{1}$ \\
\hline 2 & 0 & 0 & 1 & $B_{2}$ & $\varpi_{4}$ \\
\hline 1 & 0 & 1 & 0 & $C_{3}\left(a_{1}\right)$ & $\varpi_{3}$ \\
\hline 0 & 2 & 0 & 2 & $F_{4}\left(a_{2}\right)$ & $\varpi_{2}$ \\
\hline 2 & 2 & 0 & 2 & $F_{4}\left(a_{1}\right)$ & $\varpi_{4}$ \\
\hline
\end{tabular}

\begin{tabular}{|c|c|c|}
\hline \multicolumn{3}{|c|}{$E_{6}$} \\
\hline$\bullet$ : $\bullet$ & Bala-Carter & Sign rep \\
\hline $\begin{array}{lllll}0 & 0 & 0 & 0 & 0 \\
& & 2 & & \end{array}$ & $A_{2}$ & $\varpi_{2}$ \\
\hline $\begin{array}{lllll}2 & 0 & 2 & 0 & 2 \\
& & 0 & & \end{array}$ & $E_{6}\left(a_{3}\right)$ & $\varpi_{4}$ \\
\hline
\end{tabular}




\begin{tabular}{|c|c|c|}
\hline \multicolumn{3}{|c|}{$E_{7}$} \\
\hline$\bullet$ ? $\bullet \bullet$ & Bala-Carter & Sign rep \\
\hline $\begin{array}{llllll}2 & 0 & 0 & 0 & 0 & 0 \\
& & 0 & & & \end{array}$ & $A_{2}$ & $\varpi_{1}$ \\
\hline $\begin{array}{llllll}1 & 0 & 0 & 0 & 1 & 0 \\
& & 0 & & & \end{array}$ & $A_{2}+A_{1}$ & $\varpi_{1}, \varpi_{6}$ \\
\hline 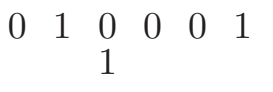 & $D_{4}\left(a_{1}\right)+A_{1}$ & $\varpi_{3}, \varpi_{2}-\varpi_{7}$ \\
\hline $\begin{array}{llllll}0 & 0 & 1 & 0 & 1 & 0\end{array}$ & $A_{3}+A_{2}$ & $\varpi_{4}, \varpi_{6}$ \\
\hline $\begin{array}{llllll}2 & 0 & 0 & 0 & 2 & 0 \\
& & & 0 & & \\
\end{array}$ & $A_{4}$ & $\varpi_{1}$ \\
\hline $\begin{array}{llllll}1 & 0 & 1 & 0 & 1 & 0 \\
& & 0 & & & \end{array}$ & $A_{4}+A_{1}$ & $\varpi_{1}, \varpi_{4}$ \\
\hline $\begin{array}{llllll}2 & 0 & 1 & 0 & 1 & 0 \\
& & & 0 & & \\
\end{array}$ & $D_{5}\left(a_{1}\right)$ & $\varpi_{4}, \varpi_{6}$ \\
\hline 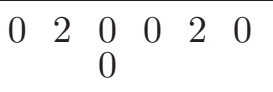 & $E_{6}\left(a_{3}\right)$ & $\varpi_{3}$ \\
\hline $\begin{array}{llllll}2 & 0 & 2 & 0 & 0 & 2 \\
& & & 0 & & \\
\end{array}$ & $E_{7}\left(a_{4}\right)$ & $\varpi_{4}$ \\
\hline $\begin{array}{llllll}2 & 0 & 2 & 0 & 2 & 0 \\
& & & 0 & & \\
\end{array}$ & $E_{6}\left(a_{1}\right)$ & $\varpi_{1}$ \\
\hline $\begin{array}{llllll}2 & 0 & 2 & 0 & 2 & 2 \\
& & & 0 & & \\
\end{array}$ & $E_{7}\left(a_{3}\right)$ & $\varpi_{4}$ \\
\hline
\end{tabular}




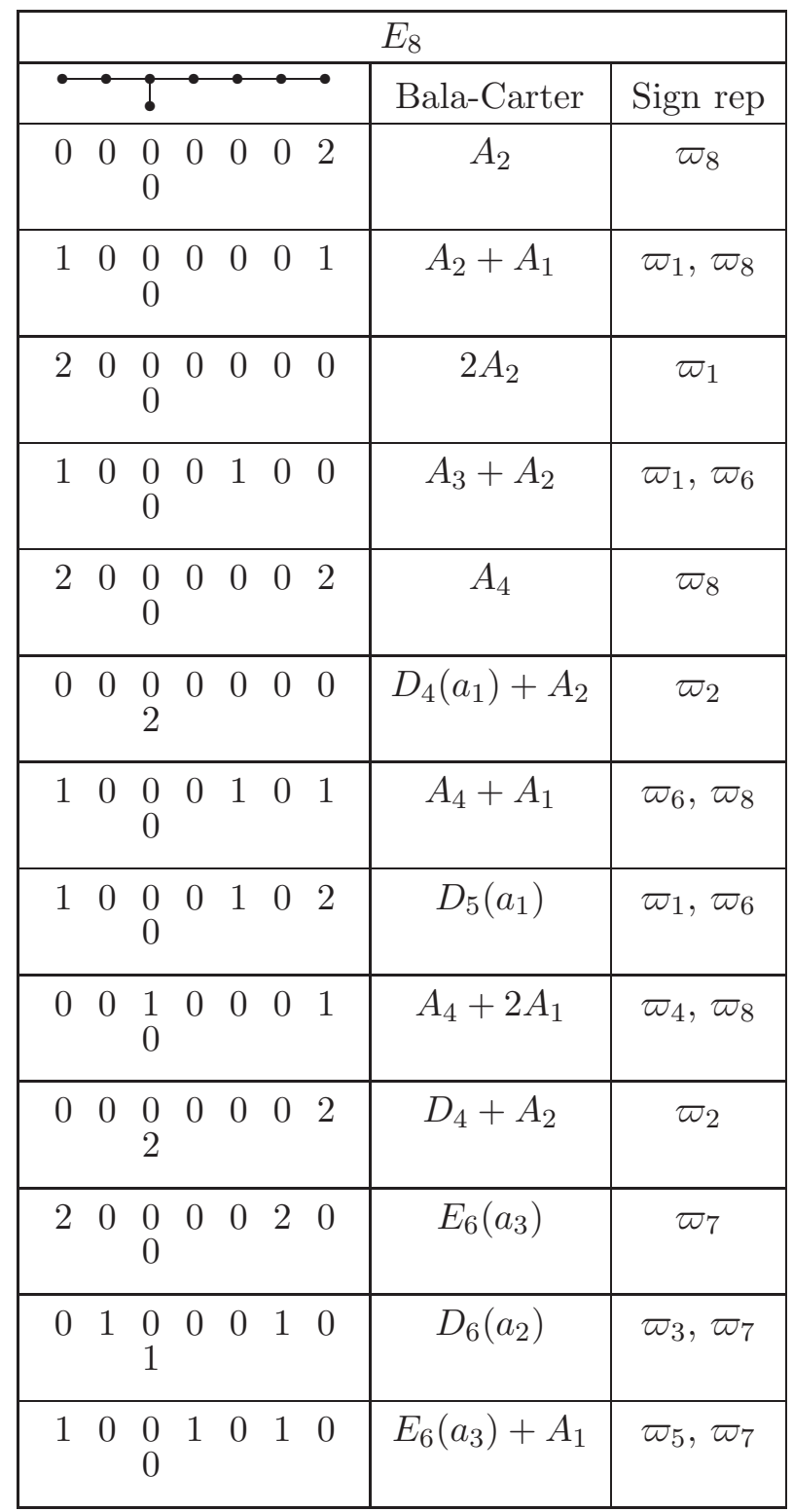




\begin{tabular}{|c|c|c|}
\hline \multicolumn{3}{|c|}{$E_{8}$} \\
\hline$\bullet ? \bullet \bullet \bullet$ & Bala-Carter & Sign rep \\
\hline $\begin{array}{lllllll}0 & 1 & 0 & 0 & 0 & 1 & 2 \\
& & 1 & & & & \end{array}$ & $D_{6}\left(a_{1}\right)$ & $\varpi_{2}, \varpi_{3}$ \\
\hline $\begin{array}{lllllll}0 & 0 & 1 & 0 & 1 & 0 & 2 \\
& & 0 & & & & \end{array}$ & $E_{7}\left(a_{4}\right)$ & $\varpi_{4}, \varpi_{6}$ \\
\hline $\begin{array}{lllllll}2 & 0 & 0 & 0 & 2 & 0 & 2 \\
& & 0 & & & & \end{array}$ & $E_{6}\left(a_{1}\right)$ & $\varpi_{8}$ \\
\hline $\begin{array}{lllllll}0 & 0 & 0 & 2 & 0 & 0 & 2 \\
& & 0 & & & & \end{array}$ & $D_{5}+A_{2}$ & $\varpi_{5}$ \\
\hline $\begin{array}{lllllll}1 & 0 & 1 & 0 & 1 & 0 & 1 \\
& & 0 & & & & \end{array}$ & $D_{7}\left(a_{2}\right)$ & $\varpi_{1}, \varpi_{4}$ \\
\hline $\begin{array}{lllllll}1 & 0 & 1 & 0 & 1 & 0 & 2 \\
& & 0 & & & & \end{array}$ & $E_{6}\left(a_{1}\right)+A_{1}$ & $\varpi_{8}$ \\
\hline $\begin{array}{lllllll}2 & 0 & 1 & 0 & 1 & 0 & 2 \\
& & 0 & & & & \end{array}$ & $E_{7}\left(a_{3}\right)$ & $\varpi_{4}, \varpi_{6}$ \\
\hline $\begin{array}{lllllll}2 & 0 & 0 & 2 & 0 & 0 & 2 \\
& & 0 & & & & \end{array}$ & $D_{7}\left(a_{1}\right)$ & $\varpi_{5}$ \\
\hline $\begin{array}{lllllll}2 & 0 & 2 & 0 & 0 & 2 & 0 \\
& & 0 & & & & \end{array}$ & $E_{8}\left(a_{5}\right)$ & $\varpi_{4}, \varpi_{7}$ \\
\hline $\begin{array}{lllllll}2 & 0 & 2 & 0 & 0 & 2 & 2 \\
& & & 0 & & & \end{array}$ & $E_{8}\left(b_{4}\right)$ & $\varpi_{4}$ \\
\hline $\begin{array}{lllllll}2 & 0 & 2 & 0 & 2 & 0 & 2 \\
& & 0 & & & & \end{array}$ & $E_{8}\left(a_{4}\right)$ & $\varpi_{4}, \varpi_{8}$ \\
\hline $\begin{array}{lllllll}2 & 0 & 2 & 0 & 2 & 2 & 2 \\
& & 0 & & & & \end{array}$ & $E_{8}\left(a_{3}\right)$ & $\varpi_{4}$ \\
\hline
\end{tabular}


Sign and standard representations of $A(e)$ when $A(e) \simeq S_{3}$

\begin{tabular}{|c|c|c|c|}
\hline \multicolumn{4}{|c|}{$A(e) \simeq S_{3}$} \\
\hline Group & Orbit & Sign & Standard \\
\hline$G_{2}$ & $G_{2}\left(a_{1}\right)$ & $\varpi_{2}$ & $\varpi_{1}$ \\
$E_{6}$ & $D_{4}\left(a_{1}\right)$ & $\varpi_{4}$ & $\varpi_{2}$ \\
$E_{7}$ & $D_{4}\left(a_{1}\right)$ & $\varpi_{3}$ & $\varpi_{1}$ \\
$E_{7}$ & $E_{7}\left(a_{5}\right)$ & $\varpi_{4}$ & $\varpi_{2}-\varpi_{7}$ \\
$E_{8}$ & $D_{4}\left(a_{1}\right)$ & $\varpi_{7}$ & $\varpi_{8}$ \\
$E_{8}$ & $D_{4}\left(a_{1}\right)+A_{1}$ & $\varpi_{2}, \varpi_{7}$ & $\varpi_{8}$ \\
$E_{8}$ & $E_{7}\left(a_{5}\right)$ & $\varpi_{4}, \varpi_{6}$ & $\varpi_{2}$ \\
$E_{8}$ & $E_{8}\left(b_{6}\right)$ & $\varpi_{4}, \varpi_{8}$ & $\varpi_{2}$ \\
$E_{8}$ & $E_{8}\left(a_{6}\right)$ & $\varpi_{4}, \varpi_{7}$ & $\varpi_{2}, \varpi_{8}$ \\
$E_{8}$ & $E_{8}\left(b_{5}\right)$ & $\varpi_{4}$ & $\varpi_{2}$ \\
\hline
\end{tabular}

Character tables when $A(e) \simeq S_{4}, S_{5}$

\begin{tabular}{|c|c|c|c|c|}
\hline \multicolumn{5}{|c|}{$F_{4}\left(a_{3}\right)$} \\
\hline Conjugacy class & $\varpi_{1}$ & $\varpi_{2}$ & $\varpi_{3}$ & $\varpi_{4}$ \\
\hline$F_{4}\left(a_{3}\right)$ & 2 & 1 & 3 & 3 \\
$A_{3}+\tilde{A}_{1}$ & 0 & -1 & 1 & -1 \\
$A_{2}+\tilde{A}_{2}$ & -1 & 1 & 0 & 0 \\
$B_{4}\left(a_{1}\right)$ & 2 & 1 & -1 & -1 \\
$A_{1}+C_{3}\left(a_{1}\right)$ & 0 & -1 & -1 & 1 \\
\hline
\end{tabular}

\begin{tabular}{|c|c|c|c|c|c|c|}
\hline \multicolumn{6}{|c|}{$E_{8}\left(a_{7}\right)$} \\
\hline Conjugacy class & $\varpi_{8}$ & $\varpi_{6}$ & $\varpi_{5}$ & $\varpi_{7}$ & $\varpi_{1}$ & $\varpi_{2}$ \\
\hline$E_{8}\left(a_{7}\right)$ & 4 & 4 & 1 & 6 & 5 & 5 \\
$A_{5}+A_{2}+A_{1}$ & -1 & 1 & -1 & 0 & 1 & -1 \\
$2 A_{4}$ & -1 & -1 & 1 & 1 & 0 & 0 \\
$D_{5}\left(a_{1}\right)+A_{3}$ & 0 & 0 & -1 & 0 & -1 & 1 \\
$D_{8}\left(a_{5}\right)$ & 0 & 0 & 1 & -2 & 1 & 1 \\
$E_{7}\left(a_{5}\right)+A_{1}$ & 2 & -2 & -1 & 0 & 1 & -1 \\
$E_{6}\left(a_{3}\right)+A_{2}$ & 1 & 1 & 1 & 0 & -1 & -1 \\
\hline
\end{tabular}




\section{Lifting in the Classical Groups}

In this section we prove Theorem 1.1 in the classical groups.

\subsection{Types $B, C, D$}

We first handle the case relative to a group that is symplectic or special orthogonal. Fix $\epsilon \in\{0,1\}$. In what follows, all congruences are modulo 2. Let $V$ be a complex vector space of dimension $N$ with a non-degenerate bilinear form $\phi: V \times V \rightarrow \mathbf{C}$ satisfying $\phi(v, w)=(-1)^{\epsilon} \phi(w, v)$ for $v, w \in V$. Let $H$ be the subgroup of $G L(V)$ preserving the form $\phi$, and let $G$ be its connected component (so $G$ is not assumed to be simply-connected in this section). Set $n=\lfloor N / 2\rfloor$. Then $G$ is of type $B_{n}$ when $\epsilon=0, N$ odd; of type $D_{n}$ when $\epsilon=0, N$ even; and of type $C_{n}$ when $\epsilon=1, N$ even.

Let $e$ be a nilpotent element in the Lie algebra of one of these groups with corresponding partition $\lambda:=\left(\lambda_{1} \geq \lambda_{2} \geq \ldots\right)$ of $N$. We first recall a description of a basis of the component groups $A^{\prime}(e):=Z_{H}(e) / Z_{H}^{\circ}(e)$ and $A(e):=Z_{G}(e) / Z_{G}^{\circ}(e)$. These are elementary abelian 2-groups with a natural injective map of $A(e)$ into $A^{\prime}(e)$.

Let

$$
\mathcal{B}(\lambda):=\left\{j \in \mathbf{N} \mid \lambda_{j}>\lambda_{j+1} \text { and } \lambda_{j} \not \equiv \epsilon\right\}
$$

Fix a normalized basis of $V$ with respect to $e$ as discussed in [5]. The basis consists of $v_{i, j}$ for $1 \leq i \leq \lambda_{j}$ and the action of $e$ is given by $e . v_{i, j}=v_{i-1, j}$ for $i>1$ and $e \cdot v_{1, j}=0$ For $k \in \mathcal{B}(\lambda)$, define an element $b_{k} \in H$ by $b_{k} \cdot v_{i, j}=$ $v_{i, j}$ when $j \neq k$ and $b_{k} \cdot v_{i, k}=-v_{i, k}$. Then $b_{k} \in Z_{H}(e)$ and the images of all the $b_{k}$ in $A^{\prime}(e)$ give a basis of $A^{\prime}(e)$ over $\mathbf{F}_{2}$. If $\epsilon=1$, then $A(e)=A^{\prime}(e)$. If $\epsilon=0$, then $A(e)$ is the subgroup of $A^{\prime}(e)$ of index two given by

$$
\left\{\sum a_{j} b_{j} \mid a_{j} \in \mathbf{F}_{2} \text { and } \sum a_{j}=0\right\}
$$

where we also use $b_{j}$ for its image in $A(e)$.

Let $p$ (respectively, $q$ ) be the largest even (respectively, odd) part of $\lambda$. Let

$$
E=\{p, p-2, \ldots, 4,2\} \text { and }
$$




$$
O=\{q, q-2, \ldots, 5,3\}
$$

For $s \in E \cup O$ define the subspace $F_{s}$ spanned by the vectors $v_{i, j}$ satisfying

$$
\lambda_{j}+2-2 i \geq s
$$

The $F_{s}$ are isotropic and satisfy $F_{s} \subset F_{s^{\prime}}$ whenever $s^{\prime} \leq s$. The subgroup of $G$ which fixes this partial isotropic flag is a parabolic subgroup $P$ of $G$. The dimension of $F_{s}$ equals $\sigma(s)$ where

$$
\sigma(s):=\sum_{\lambda_{j} \geq s}\left(\left\lfloor\frac{\lambda_{j}-s}{2}\right\rfloor+1\right) .
$$

Let $T$ be a maximal torus of $G$ such that the $v_{i, j}$ with $\lambda_{j}+1-2 i>0$ are weight vectors for $T$. Then $T \subset P$. Choose a Borel subgroup $B$ of $G$ with $T \subset B \subset P$. Next let $\gamma\left(\mathbf{C}^{*}\right) \subset T$ be the one-parameter subgroup of $G$ given by

$$
\gamma(z) \cdot v_{i, j}=z^{\lambda_{j}+1-2 i} v_{i, j}
$$

for $z \in \mathbf{C}^{*}$. Then $L:=Z_{G}(\gamma)$ is a Levi subgroup of $P$, which contains $T$. Now, $\gamma$ defines a cocharacter associated to $e$ and therefore $Z_{G}(e, \gamma)$ defines a Levi subgroup of $Z_{G}(e)$. Moreover, $P$ is the Jacobson-Morozov parabolic associated to $e$ and $\gamma$.

For $s \in E \cup O \cup\{1\}$, define $L_{s}$ to be the subgroup of $G$ that preserves the subspace spanned by the vectors

$$
v_{i, j} \text { with } \lambda_{j}+2-2 i=s
$$

and is the identity on all other $v_{i, j}$. Then when $s>1, L_{s} \simeq G L_{d_{s}}(\mathbf{C})$ where

$$
d_{s}=\sigma(s)-\sigma(s+1)=\#\left\{j \mid \lambda_{j} \geq s, \lambda_{j} \equiv s\right\}
$$

On the other hand, $L_{1}$ is of the same type as $G$, but acting on a vector space of dimension $\#\left\{j \mid \lambda_{j} \equiv 1\right\}$. Then

$$
L \simeq \prod_{s \in E \cup O \cup\{1\}} L_{s}
$$


For $s \in E \cup O$, define a character $\chi_{s}$ of $T$ such that for $t \in T$, we have

$$
\chi_{s}(t)=\prod_{\lambda_{j}+2-2 i=s} t_{i, j},
$$

where $t . v_{i, j}=t_{i, j} v_{i, j}$. This character gives rise to the one-dimensional representation of $L$ (and $P$ ) which is trivial on $L_{k}$ for $k \neq s$ and yields the determinant representation of $L_{s} \simeq G L_{d_{s}}(\mathbf{C})$. Let $\Xi$ be the elements of $E \cup O$ such that $\sigma(s)$ is not equal to $n$ in type $B_{n}$ and is not equal to $n$ or $n-1$ in type $D_{n}$. Then for $s \in \Xi$, we have that $\varpi_{\sigma(s)}$ is the weight of a character of $T$, relative to our choice of $B$. Moreover, $\chi_{s}=\varpi_{\sigma(s)}$ for $s=\max (E \cup O)$ and $\chi_{s}=\varpi_{\sigma(s)}-\varpi_{\sigma(s+1)}$, otherwise. Note that $\chi_{s}$ is conjugate under $W$ to $\varpi_{d_{s}}$.

For $s \in \Xi$ let $\pi_{s}$ be the projection of $L$ onto $L_{s}$. Then $\pi_{s}\left(Z_{H}(e, \gamma)\right)$ equals

$$
\begin{gathered}
\prod_{m \equiv s, m \geq s} S p_{r_{m}}(\mathbf{C}) \text { when } s \equiv \epsilon \text { and } \\
\prod_{m \equiv s, m \geq s} O_{r_{m}}(\mathbf{C}) \text { when } s \not \equiv \epsilon
\end{gathered}
$$

where $r_{m}$ is the number of parts of $\lambda$ equal to $m$. Each factor on the right above sits in $L_{s}$ as a subgroup preserving the vectors $v_{i, j}$ with $\lambda_{j}+2-2 i=s$ and $m=\lambda_{j}$. It follows that $\pi_{s}\left(Z_{H}^{\circ}(e, \gamma)\right)$ consists of matrices of determinant one, sitting in $L_{s} \simeq G L_{d_{s}}(\mathbf{C})$. Consequently, each one-dimensional representation of $L_{s}$, and hence each $\chi_{s}$, when viewed as a representation of $P$ trivial on $U_{P}$, is trivial on $Z_{H}^{\circ}(e)=Z_{G}^{\circ}(e)$ and thus descends to a representation of $A(e)$.

In type $C_{n}$, we add a part equal to zero as the last part of $\lambda$ and then in all types we define $k_{\max }$ to be the largest element of $\mathcal{B}(\lambda)$ (so $\lambda_{k_{\max }}=0$ in type $\left.C_{n}\right)$. Define $\widetilde{\mathcal{B}}(\lambda)=\mathcal{B}(\lambda)-\left\{k_{\max }\right\}$. For $k \in \widetilde{\mathcal{B}}(\lambda)$, set

$$
\tilde{b}_{k}=b_{k} b_{k^{\prime}}
$$

where $k^{\prime}$ is minimal for the property that $k^{\prime} \in \mathcal{B}(\lambda)$ and $k<k^{\prime}$ and we set $b_{k_{\max }}$ to be the identity in type $C$. Then the images of the $\tilde{b}_{k}$, for $k \in \widetilde{\mathcal{B}}(\lambda)$, give a basis of $A(e)$ over $\mathbf{F}_{2}$. 
Let $\mathcal{S} \subset \widetilde{\mathcal{B}}(\lambda)$ and denote by $\bar{\chi}_{\mathcal{S}}$ the one-dimensional representation of $A(e)$ given by

$$
\begin{aligned}
& \bar{\chi}_{\mathcal{S}}\left(\tilde{b}_{k}\right)=-1 \text { if } k \in \mathcal{S} \\
& \bar{\chi}_{\mathcal{S}}\left(\tilde{b}_{k}\right)=1 \text { if } k \notin \mathcal{S},
\end{aligned}
$$

allowing $\tilde{b}_{k}$ to also stand for its image in $A(e)$. In this way, the characters of $A(e)$ are parametrized by the subsets of $\widetilde{B}(\lambda)$.

Proposition 3.1. Let e be a nilpotent element in types $B_{n}, C_{n}, D_{n}$ with partition $\lambda$. Given $\mathcal{S} \subset \widetilde{B}(\lambda)$, let

$$
\chi_{\mathcal{S}}:=\sum_{j \in \mathcal{S}} \chi_{\lambda_{j}} .
$$

Then $\chi_{\mathcal{S}}$ is a lifting to $P$ of $\bar{\chi}_{\mathcal{S}}$. This lift is of minimal length.

Proof. By the above discussion, each $\chi_{s}$ for $s \in \Xi$ descends to a character of $A(e)$. Next, it is clear from (3.1) that for $s \equiv \epsilon$ that $\chi_{s}$ is trivial on $A(e)$. Also this holds for $s \leq \lambda_{k_{\max }}$. For other $s$, we have $\chi_{s}=\chi_{\lambda_{k}}$ where $\lambda_{k^{\prime}}<s \leq \lambda_{k}$ for $k^{\prime}, k \in \mathcal{B}(\lambda)$. Moreover for $j \in \widetilde{B}(\lambda)$ it is clear that $\chi_{\lambda_{j}}$ restricts to $\bar{\chi}_{\{j\}}$. This completes the proof except for the claim of minimal length.

In the usual inner produce on weights of $G$, the $\chi_{s}$ are mutually orthogonal. They also generate the lattice of one-dimensional characters of $L$, together with possibly one additional weight (in type $B$, when there is exactly one odd $\lambda_{j}$ ) and up to two additional weights (in type $D$, when there are at most two odd $\lambda_{j}$ 's). These additional weights descend to $A(e)$ and are trivial (this will also follow from results in the next section). The additional weight(s) can be chosen to be orthogonal to all $\chi_{s}$ (and to each other). The orthogonality of these basis elements of the lattice then implies that the $\chi_{\mathcal{S}}$ are minimal lifts.

\subsection{Liftings for simply-connected type in the classical groups}

It remains to treat the case of the spin groups, which are 2 -fold covers of the groups $G$ from the previous section in types $B$ and $D$. Fix $G$ of type $B_{n}(n \geq 2)$ or $D_{n}(n \geq 3)$ from the previous section. Let $\tilde{G}$ denote the 
spin group covering of $G$ with isogeny $f: \tilde{G} \rightarrow G$. Let $\tilde{L}=f^{-1}(L)$ and $\tilde{P}=f^{-1}(P)$. The kernel of $f$ is a group of order 2 generated by an element $c$ in the center of $\tilde{G}$. Clearly, $f\left(\tilde{G}_{e}\right)=G_{e}$ and $f\left(\tilde{G}_{e}^{\circ}\right)=G_{e}^{\circ}$ and so $f$ induces a surjection of

$$
A_{\tilde{G}}(e):=\tilde{G}_{e} / \tilde{G}_{e}^{\circ}
$$

onto $A_{G}(e)$ with a kernel that is either trivial, or non-trivial and generated by the image of $c$.

From [7] (see also [10]), the kernel is non-trivial if the odd parts of the partition $\lambda$ of $e$ occur with multiplicity one. To that end, let $e$ be a nilpotent element with partition $\lambda$ such that $r_{i} \in\{0,1\}$ when $i$ is odd. Note that in type $D$ such an element $e$ could be very even. Retain the notation of the previous section. Let $m=|\widetilde{\mathcal{B}}(\lambda)|+1$. Then $\left|A_{\tilde{G}}(e)\right|=2^{m}$.

Proposition 3.2. In type $B_{n}$ the representation $V_{\varpi_{n}}$ of $\tilde{L}$ of highest weight $\varpi_{n}$ has dimension $2^{\frac{m-1}{2}}$. When lifted to $\tilde{P}$, it descends to an irreducible representation of $A_{\tilde{G}}(e)$. Together with the $2^{m-1}$ characters of $P$ in Proposition 3.1 that descend to $A_{G}(e)$, these form a complete set of irreducible representations of $A_{\tilde{G}}(e)$.

Proof. Let $\Phi_{J}$ be of type

$$
A_{a_{1}} \times \cdots \times A_{a_{k}} \times B_{q}
$$

where $2 q+1$ is the sum of the odd parts of $\lambda$ and the even parts of $\lambda$ are

$$
\left[a_{1}+1, a_{1}+1, \ldots, a_{k}+1, a_{k}+1\right] \text {. }
$$

Assume that the factors of $\Phi_{J}$ sit in $G$ in the order written above.

Take $e_{1} \in \mathfrak{g}_{J}$ so that $e_{1}$ is regular in the type $A$ factors and has partition in the $B_{l}$ factor consisting of the odd parts of $\lambda$. Then $e_{1}$ is distinguished in the Levi subalgebra $\mathfrak{g}_{J}$. The Dynkin element $h_{1}$ in $\mathfrak{g}_{J}$ is given by (in the standard basis for coweights)

$$
(a_{1}, a_{1}-2, \ldots,-a_{1}+2,-a_{1}, \ldots a_{k}, a_{k}-2, \ldots,-a_{k}+2,-a_{k}, y_{1}, \ldots, y_{l}, \overbrace{0, \ldots, 0}^{\frac{m-1}{2}})
$$

where $y_{i}$ are positive even integers listed in nonincreasing order. We can conjugate $h_{1}$ to be dominant using an element $w \in W$ which flips all signs 
of the negative elements in $h_{1}$ and then permutes the nonzero elements of $h_{1}$ into nonincreasing order.

We can now apply Proposition 1.2 to show that $V_{\varpi_{n}}$ is trivial on $\tilde{G}_{e}^{\circ}$. First observe that $V_{\varpi_{n}}$ restricts to give the spin representation of $f^{-1}\left(L_{1}\right)$, a spin group of type $B_{\frac{m-1}{2}}$. Hence the representation is $2^{\frac{m-1}{2}}$-dimensional and all weights are $W_{L}$-conjugate. Therefore, it is enough to check that the weight $\varpi_{n}$ is trivial on a maximal torus of $\tilde{G}_{e}^{\circ}$. Next, write $\varpi_{n}$ in the standard basis for weights in $B_{n}$ as $\varpi_{n}=\left(\frac{1}{2}, \ldots, \frac{1}{2}\right)$. Then $w^{-1}\left(\varpi_{n}\right)$ equals

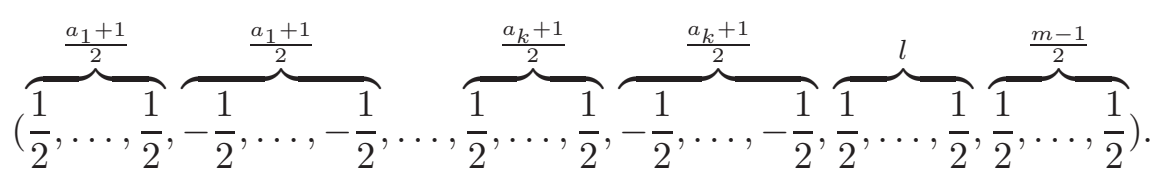

Twice this weight lies $\mathcal{L}_{J}$. Hence $V_{\varpi_{n}}$ descends to a representation of $A_{\tilde{G}}(e)$.

Now the weights of $V_{\varpi_{n}}$ are not weights of $G$; hence they take the value -1 on the central element $c$ of $\tilde{G}$. So $c$ acts by -1 on $V_{\varpi_{n}}$. Now for $x \in A_{\tilde{G}}(e)$ with $x \neq 1, c$, we have that $x$ and $x c$ are conjugate in $A_{\tilde{G}}(e)$ by [7] (see also [11, Proposition 26]). It follows that

$$
\operatorname{tr}\left(x, V_{\varpi_{n}}\right)=\operatorname{tr}\left(x c, V_{\varpi_{n}}\right)=-\operatorname{tr}\left(x, V_{\varpi_{n}}\right)
$$

and so $\operatorname{tr}\left(x, V_{\varpi_{n}}\right)=0$ for $x \neq 1, c$. Therefore the inner product of the character of $V_{\varpi_{n}}$ with itself is computed just on $1, c \in A_{\tilde{G}}(e)$, yielding

$$
\frac{1}{\left|A_{\tilde{G}}(e)\right|}\left(\left(2^{\frac{m-1}{2}}\right)^{2}+\left(-2^{\frac{m-1}{2}}\right)^{2}\right)=\frac{1}{2^{m}} 2^{m}=1,
$$

which shows that $V_{\varpi_{n}}$ is an irreducible representation of $A_{\tilde{G}}(e)$. Finally, the sum of the squares of the dimensions of the distinct irreducible representations of $A_{\tilde{G}}(e)$ that we have constructed equals

$$
1 \cdot\left(2^{\frac{m-1}{2}}\right)^{2}+2^{m-1} \cdot\left(1^{2}\right)=2^{m-1}+2^{m-1}=2^{m},
$$

which shows that we have found them all.

To obtain a weight of minimal length for the extra representation, we can subtract off the fundamental weight for the the largest nonzero node $i$ of the weighted Dynkin diagram with $i \leq n-1$, if such a node exists. 
Proposition 3.3. In type $D_{n}$ when $m \geq 2$, the representations $V_{\varpi_{n}}, V_{\varpi_{n-1}}$ of $\tilde{P}$ are both of dimension $2^{\frac{m}{2}-1}$ and descend to give irreducible representations of $A_{\tilde{G}}(e)$. Together with the $2^{m-1}$ irreducible representations of $P$ in Theorem 3.1 which descend to $A_{G}(e)$, these form a complete set of irreducible representations of $A_{\tilde{G}}(e)$.

Proof. In this case $L_{1}$ is of type $D_{\frac{m}{2}}$ and $V_{\varpi_{n}}, V_{\varpi_{n-1}}$ restrict to give the two half-spin representations of $f^{-1}\left(L_{1}\right)$, which are of dimension $2^{\frac{m}{2}-1}$. In case $m=2$, we think of $D_{1}$ as the center of $\tilde{G}$ and the half-spin representations are one-dimensional representations which are non-trivial on $c$.

As in type $B$, the weights of these representations are $W_{L}$-conjugate and so a similar argument yields that $\tilde{G}_{e}^{\circ}$ acts trivially and that $c$ acts by -1 . Next, the center of $A_{\tilde{G}}(e)$ coincides with the image of the center of $\tilde{G}$ and is of order 4. Moreover for $x \in A_{\tilde{G}}(e)$, with $x$ not in the center of $A_{\tilde{G}}(e)$, the elements $x$ and $x c$ are conjugate in $A_{\tilde{G}}(e)$ [7] (see also [11, §4]). Thus the character of these representations are zero away from the four central elements of $A_{\tilde{G}}(e)$ and the images of central elements of $\tilde{G}$ act by scalars since $V_{\varpi_{n-1}}$ and $V_{\varpi_{n}}$ are irreducible representations of $f^{-1}(L)$. The scalars must be roots of unity and so the inner product of the character of either representation with itself is equal to 1 For the last statement we have the sum of the squares of the known irreducibles is

$$
2\left(2^{\frac{m}{2}-1}\right)^{2}+1^{2}\left(2^{m-1}\right)=2^{m-1}+2^{m-1}=2^{m}
$$

as desired.

For the case where $m=1$, that is, when $e$ is very even, either $V_{\varpi_{n-1}}$ or $V_{\varpi_{n}}$ will give the desired one-dimensional representation, depending on which node of the weighted Dynkin diagram is nonzero.

To obtain a weight of minimal length for the two extra representations, we can subtract off the fundamental weight for the the largest nonzero node $i$ of the weighted Dynkin diagram with $i \leq n-2$, if such a node exists.

\subsection{Type $A$}

Let $G$ be the special linear group $S L_{l}(\mathbf{C})$. Let $\lambda:=\left(\lambda_{1} \geq \lambda_{2} \geq \cdots \geq \lambda_{k}\right)$ be a partition of $l$ and let $d$ be the greatest common divisor of $\lambda_{1}, \lambda_{2}, \ldots, \lambda_{k}$. 
Let $e$ be a nilpotent element corresponding to $\lambda$. Then $A(e) \simeq \mathbb{Z} / d \mathbb{Z}$. Let $q=\frac{l}{d}$. It is easy to check that for $\varpi_{j q}$ fulfill the conditions of Theorem 1.1 for $0 \leq j \leq d-1$ and that these weights are of minimal length.

\section{Applications}

\subsection{Special pieces}

We refer to [8] for definitions. Assume here that $G$ is of adjoint type. Let $\mathcal{O}_{\text {sp }}=\mathcal{O} \cup \mathcal{O}^{\prime}$ be a special piece in an exceptional group such that $A(e) \simeq S_{2}$ for $e$ in the special orbit $\mathcal{O}$. Let $\lambda$ be a minimal lift to $P$ of the sign representation of $A(e)$ as in Theorem 1.1. Let $\mu$ be the dominant weight that is $W$-conjugate to $\lambda$ and let $V$ be a representation of $G$ of highest weight $\mu$. Consider the $G$-orbit $Z$ of $(e, v) \in \mathfrak{g} \oplus V$, where $v \in V$ is a weight vector of weight $\lambda$. Then the stabilizer of $(e, v)$ in $G$ equals $G_{e}^{\circ}$ and thus $Z \simeq G / G_{e}^{\circ}$. Let $\bar{Z}$ be the closure of $Z$ in $\mathfrak{g} \oplus V$.

From the tables in [3], one observes that there is a transverse slice $\mathcal{S}$ in $\overline{\mathcal{O}}$ to a point $e^{\prime} \in \mathcal{O}^{\prime}$ that is isomorphic to the closure of the minimal orbit $X_{\text {min }}$ in a symplectic group of type $C_{n}$. Let $p: \bar{Z} \rightarrow \overline{\mathcal{O}}$ be the restriction to $\bar{Z}$ of the projection of $\mathfrak{g} \oplus V$ onto the first factor.

In work in progress with Fu, Juteau, and Levy, we checked that

Proposition 4.1. The pre-image $p^{-1}(\mathcal{S})$ is isomorphic to $\mathbf{C}^{2 n}$ and $p^{-1}(\mathcal{S})$ identifies with the affinization of the universal cover of $X_{\text {min }}$.

The proof amounts to checking that when $V$ is restricted to a certain reductive centralizer of $e^{\prime}$ (which happens to contains a simple factor of type $C_{n}$ ), the vector $v$ lies in a subrepresentation isomorphic to the defining representation of $S p_{2 n}(\mathbf{C})$. A consequence of Proposition 4.1 is that $p^{-1}\left(\mathcal{O}_{\mathrm{sp}}\right)$ is smooth and satisfies the desired properties of $C^{\dagger}$ from [8, §0.6]. A statement similar to Proposition 4.1 is expected to hold for the other special pieces in the exceptional groups.

\subsection{Module structure of sections of local systems}

In [6] Jantzen gives an exposition of results in McGovern [9] and Graham [4], results we propose to generalize, and we refer there for definitions. Let 
$\tilde{\mathcal{O}}:=G / G_{e}^{\circ}$ denote the universal cover of $\mathcal{O}$, which carries a left action of $G$ and a right action of $A(e)$. The $\mathbf{C}^{*}$-action on $\mathcal{O}$ lifts compatibly to $\tilde{\mathcal{O}}$ so that the regular functions $\mathbf{C}[\tilde{\mathcal{O}}]_{(n)}$ of degree $n$ on $\tilde{\mathcal{O}}$ are a $G \times A(e)$-module. Let $\hat{A}(e)$ refer to the isomorphism classes of irreducible representations of $A(e)$. We can write

$$
\mathbf{C}[\tilde{\mathcal{O}}]_{(n)} \simeq \bigoplus_{(\pi, V) \in \hat{A}(e)} V \otimes \Gamma_{V}^{n}
$$

where $\Gamma_{V}^{n}$ are the degree $n$ sections of the vector bundle $G \times{ }^{G_{e}} V \rightarrow \mathcal{O}$ corresponding to a lift of $V$ to $G_{e}$, which is trivial on $G_{e}^{\circ}$. We note that the grading is such that $\Gamma_{\text {trivial }}^{1}=0$ and $\Gamma_{\text {trivial }}^{2}$ is isomorphic to the adjoint representation.

It is expected that Theorem $1.1 \mathrm{can}$ be used to determine $\Gamma_{V}^{n}$ as follows. Let $(\pi, V) \in \hat{A}(e)$. Let $(\widetilde{\pi}, V)$ be a lifting to $P$ as in Theorem 1.1. Let $\lambda$ be a lowest weight of $\tilde{\pi}$ so that $|\lambda|$ is minimal among lowest weights of all possible lifts of $V$. In fact $\lambda$ can always be taken to be a sum of positive roots. Let $\mathbf{C}_{\lambda}$ be the one-dimensional representation of $B$ defined by $\lambda$.

Conjecture 4.2. For $\lambda$ as above, we have

$$
\Gamma_{V}^{n} \simeq H^{0}\left(G / B, S^{(n+\langle\lambda, \chi\rangle) / 2} \mathfrak{g}_{\geq 2}^{*} \otimes \mathbf{C}_{\lambda}\right)
$$

for all $n \in \mathbb{Z}$ and

$$
H^{i}\left(G / B, S^{n} \mathfrak{g}_{\geq 2}^{*} \otimes \mathbf{C}_{\lambda}\right)=0
$$

for $i>0$ and for all $n$.

Here, $\langle$,$\rangle is the pairing of characters and cocharacters of T$, and $\chi \in T$ is the cocharacter associated to $e$ and $P$. It is well-known that in cases where the conjecture holds that there are explicit formulas for the multiplicity of a highest weight representation of $G$ in $\Gamma_{V}^{n}$ using Lusztig's $q$-analogue of Kostant's weight multiplicity.

We are able to prove a variation of the result, and hence still compute $\Gamma_{V}^{n}$, in the following two situations: (1) when $G$ is of type $A$ and $e$ is any nilpotent element, or (2) when $e$ is even and $V$ is one-dimensional. In the variation we may have to replace $\mathfrak{g}_{\geq 2}^{*}$ by $\mathfrak{u}_{P^{\prime}}$, the Lie algebra of the unipotent radical of a parabolic subgroup $P^{\prime}$ such that $e$ belongs to the Richardson 
orbit in $\mathfrak{u}_{P^{\prime}}$. Moreover in case (2), we may have to replace $\lambda$ by a weight $\mu \in X^{*}\left(P^{\prime}\right)$ which is $W$-conjugate to $\lambda$ and dominant. The proof uses cohomological statements of the kind proved in [13] and [12]. We finish with an example that can be treated using results already in the literature.

Example 4.3. Let $G$ of type $A_{5}$ and $e$ have partition $(4,2)$. Consider the weight $\lambda=\varpi_{3}$ in Theorem 1.1 which corresponds to the non-trivial representation $V$ of $A(e) \simeq \mathbb{Z} / 2 \mathbb{Z}$. Now $2 \varpi_{3}$ equals 12321 in the basis of simple roots. For $S \subset\{1, \ldots,|\Pi|\}$, write $\mathfrak{u}_{S}$ for the Lie algebra of the unipotent radical of a parabolic subgroup containing $B$ whose maximal roots are the $-\alpha_{i}$ for $i \in S$. Now

$$
\begin{aligned}
& H^{i}\left(S^{n} \mathfrak{u}_{1,3,5}^{*} \otimes-2 \varpi_{3}\right)=H^{i}\left(S^{n-2} \mathfrak{u}_{1,3,5}^{*} \otimes-1-1-1-1-1\right) \\
& =H^{i}\left(S^{n-3} \mathfrak{u}_{2,3,5}^{*} \otimes 00-1-1-1\right) \\
& =H^{i}\left(S^{n-4} \mathfrak{u}_{2,3,4}^{*} \otimes 00-100\right) \\
& =H^{i}\left(S^{n-5} \mathfrak{u}_{2,3,4}^{*}\right)=H^{i}\left(S^{n-5} \mathfrak{u}_{1,3,5}^{*}\right)
\end{aligned}
$$

for all $i \geq 0$ and all $n$, using the main theorem in [13] five times (we only need this result for $i=0)$. By the proof of Proposition 1.4 in [4] it follows that $\Gamma_{V}^{n}=H^{0}\left(S^{(n+5) / 2}\left(\mathfrak{u}_{1,3,5}^{*}\right) \otimes-\varpi_{3}\right)$.

Similarly, $H^{i}\left(S^{(n+5) / 2}\left(\mathfrak{u}_{1,3,5}^{*}\right) \otimes-\varpi_{3}\right)=H^{i}\left(S^{(n-5) / 2}\left(\mathfrak{u}_{1,3,5}^{*}\right) \otimes \varpi_{3}\right)$ for all $i \geq 0$ and all $n$. The latter modules are known to vanish for $i>0$ and all $n$ since $\varpi_{3}$ is dominant. Hence in this case the conjecture itself holds.

\section{References}

1. D. Barbasch and D. Vogan, Unipotent representations of complex semisimple Lie groups, Ann. Math., 121 (1985), 41-110.

2. R. W. Carter, Finite groups of Lie type, Wiley Classics Library, John Wiley \& Sons Ltd., Chichester, 1993, Conjugacy classes and complex characters, Reprint of the 1985 original, A Wiley-Interscience Publication.

3. B. Fu, D. Juteau, P. Levy and E. Sommers, Generic singularities of nilpotent orbit closures, Advances in Mathematics, 305 (2017), 1-77.

4. W. A. Graham, Functions on the universal cover of the principal nilpotent orbit, Invent. Math., 108 (1992), No.1, 15-27.

5. W. H. Hesselink, Polarizations in the classical groups, Math. Z., 160 (1978), No. 3, $217-234$. 
6. J. C. Jantzen, Nilpotent orbits in representation theory, Lie theory, Progr. Math., vol. 228, Birkhäuser Boston, Boston, MA, 2004, pp. 1-211.

7. G. Lusztig, Intersection cohomology complexes on a reductive group, Invent. Math. 75 (1984), no. 2, 205-272.

8. G. Lusztig, Notes on unipotent classes, Asian J. Math., 1 (1997), 194-207.

9. W. M. McGovern, Rings of regular functions on nilpotent orbits and their covers, Invent. Math., 97 (1989), No. 1, 209-217.

10. E. Sommers, Nilpotent orbits and the affine flag manifold, Ph.D. thesis, Massachusetts Institute of Technology, 1997.

11. E. Sommers, A generalization of the Bala-Carter theorem for nilpotent orbits, Internat. Math. Res. Notices (1998), No. 11, 539-562.

12. E. Sommers, Normality of very even nilpotent varieties in $D_{2 l}$, Bull. London Math. Soc., 37 (2005), No. 3, 351-360.

13. E. Sommers, Cohomology of line bundles on the cotangent bundle of a Grassmannian, Proc. Amer. Math. Soc., 137 (2009), No. 10, 3291-3296. 